\title{
WISSENSCHAFT UND ZEITGESCHICHTE: DIE BRIEFE ERNST VON STERNS AN EDUARD MEYER (1894-1918)
}

\author{
Jürgen von Ungern-Sternberg \\ Universität Basel, Departement Altertumswissenschaften, Alte Geschichte, \\ Petersgraben 51, CH - 4051 Basel, Schweiz; j.vonungern@unibas.ch
}

18 letters are preserved which Ernst von Stern, professor of ancient history at the universities of Odessa and Halle, wrote to the eminent historian Eduard Meyer between 1894 and 1918. They deal in an interesting way with some problems of ancient history and the copyright of authors. But they are more important as testimonies on a crucial time of Russian history: the first Russian revolution in 1905 and the following years, and again for the situation during the negotiations at Brest-Litowsk in January 1918.

Keywords: Ancient history, Russian scholars of antiquity, Russian revolution in 1905, copyright in Russia, Brest-Litowsk.

\section{I}

Eduard Meyer (1855-1930) und Ernst von Stern (1859-1924) könnten sich bereits Ende der 1870er Jahre in Leipzig kennengelernt haben. Meyer hatte sich dort im Frühjahr 1879 habilitiert und lehrte ab dem Sommersemester als Privatdozent für Alte Geschichte ${ }^{1}$. Ernst von Stern war für seine altertumswissenschaftlichen Studien von 1877-1880 Mitglied des „Russischen Philologischen Seminars“ in Leipzig ${ }^{2}$. Der erste erhaltene Brief ${ }^{3}$ von Sterns datiert aber erst vom 8. August 1894 - ein Dank für die Gastfreundschaft, die er und seine Frau bei dem Ehepaar Meyer aus Anlass der Feiern zum 200-jährigen Jubiläum der Universität Halle hatten erfahren dürfen ${ }^{4}$. Meyer war im Jahre 1889 zum ersten ordentlichen Professor für Alte Geschichte in Halle ernannt worden ${ }^{5}$, von Stern war von 1884 bis 1886 Dozent, seit 1886 außerordentlicher und seit 1888 ordentlicher Professor in Odessa, wo er sich vor allem - auch durch eigene Grabungen - mit der Archäologie und Geschichte der Nordküste des Schwarzen Meeres befasste. Bei dem Jubiläum war er der offizielle Vertreter der Universität Odessa.

${ }^{1}$ Hoffmann 1990, 240. Übrigens trat Meyer 1879 auch in eine erste Beziehung zu Russland, indem er für eine in St. Petersburg im Jahre 1880 erschienene Allgemeine Geschichte der Literatur einen Beitrag zur altorientalischen Literatur schrieb. Das deutsche Manuskript ist jetzt herausgegeben von Audring 2009; vgl. den Überblick von Frolov 1991.

2 Zu seiner Biographie s. v. Ungern-Sternberg 2015, mit der früheren Literatur. Ein Schriftenverzeichnis Ernst von Sterns von W. Göber findet sich als Anhang in: Karo 1925, 96-103.

${ }^{3}$ Die Briefe Ernst von Sterns befinden sich im Archiv der Berlin-Brandenburgischen Akademie der Wissenschaften unter den Nr. 1255 und 1506. Ich danke der Leiterin Dr. Vera Enke für die freundliche Publikationserlaubnis; für Auskünfte danke ich auch Olaf Schlunke und für freundliche Hilfe hinsichtlich russischer Fragen Alexander Verlinsky. Ein Nachlass von Sterns ist nach Auskunft von Universitätsbibliothek und Universitätsarchiv Halle dort nicht befindlich. Die teilweise doppelt datierten Briefe werden hier nur nach dem gregorianischen Kalender datiert.

${ }^{4}$ Beyschlag 1895, 32 zu von Stern; 115 zur lateinischen Grußadresse der Universität Odessa. Meyers Beitrag zur gleichzeitigen Festschrift waren seine Untersuchungen zur Geschichte der Gracchen, ein Thema, das von Stern später auch vielfach beschäftigen sollte.

5 Zimmermann 1991.

(c) St. Petersburg State University, 2016 
Die solchermaßen unter glücklichen Vorzeichen hergestellte Verbindung nutzte von Stern, um in seinem nächsten Brief vom 29. Januar 1895 einen seiner „fähigsten Schüler“, den Privatdozenten Michael Mandes (1866-1934) zu empfehlen, der auf seinen „Vorschlag ein Reisestipendium auf 2 Jahre vom Ministerium erhalten" habe ${ }^{6}$ und nun zunächst nach Halle zu dem klassischen Philologen und Archäologen Carl Robert (1850-1922) und Meyer zur Weiterbildung gehen wolle. Bemerkenswert ist die klare Charakterisierung:

„Ich hoffe zuversichtlich, dass aus ihm ein tüchtiger Gelehrter werden kann, sobald er erst einige störende Eigenschaften - eine gewisse Selbstüberschätzung und einen allzu grossen Hang ins Universale zu streben, - Eigenheiten, die sich bei Kenntnis der hiesigen Verhältnisse und der Lage, aus der er sich emporgearbeitet hat, begreifen lassen, wird abgeschliffen haben“. Insgesamt bedürfe er also „mehr des Zügels als des Sporns“.

Mandes hat sich durchaus bewährt und von Stern konnte seine ersten Schriften lobend referieren ${ }^{7}$; zustimmend zitiert er ihn auch in seinem Aufsatz: Der Mauerbau in Athen und die List des Themistokles (1904).

\section{II}

Für einige Jahre setzte die Korrespondenz wieder aus; es gab aber wechselseitige Rezensionen. Meyer besprach eher kritisch von Sterns Arbeit zum Ursprung des Ephorats; andererseits fanden die Bände III - V von Meyers, Geschichte des Altertums' zwar generell das hohe Lob von Sterns, im einzelnen machte er indes zahlreiche kritische Bemerkungen ${ }^{9}$.

Wegen des themistokleischen Mauerbaus kam es aber zu einer direkten wissenschaftlichen Kontroverse mit Eduard Meyer. In einer kurzen Postkarte vom 19. Dezember 1904 „fürchtete“ von Stern zu Recht, „dass ich Sie nicht habe überzeugen können“, und in der Tat hat Meyer in seinem Aufsatz: Der Mauerbau des Themistokles ${ }^{10}$ sich umgehend kritisch mit ihm auseinandergesetzt.

Wie schon zuvor Julius Beloch ${ }^{11}$ und Bruno Keil ${ }^{12}$ hatte von Stern den Bericht des Thukydides (I 89-93) kritisiert, dass Themistokles die Ummauerung Athens im Jahre 479 v. Chr. durch eine Überlistung der widerstrebenden Spartaner durchgesetzt habe, und hatte diese mit der weiter bestehenden Notwendigkeit der Verteidigung gegen die Perser erklärt. Diesen Zweifel an der Zuverlässigkeit des von ihm hochverehrten athenischen Historikers konnte Meyer nicht hinnehmen, auch wenn er einzelne Übertreibungen zugab. Er hat sich auch durch die Argumente, die von Stern ihm daraufhin nochmals brieflich am 24. Oktober 1905 darlegte, nicht überzeugen lassen, wie seine spätere Anmerkung

${ }^{6} \mathrm{Zu}$ diesen Stipendien s. Maurer 2004.

7 Mandes 1898 [Мандес М.И. Мессенские войны и восстановление Мессении: история и традиция]; 1901 [Мандес М.И.Опыт историко-критического комментария к греческой истории Диодора], s. Stern 1898 und 1902; vgl. auch Frolov 2006 [Фролов Э. Д. Русская наука об античности: историографические очерки. 2-е изд. СПб. 2006], 323; 339-340.

${ }^{8}$ Meyer 1894.

9 Stern 1903, 312; 331 weitere Bezugnahmen auf Mandes.

10 Meyer 1905.

11 Beloch 1893, 458 n. 2.

12 Keil 1902, 282-301. 
in der, Geschichte des Altertums' zeigt, wo er ohne Namensnennung schreibt, dass er mit seinem Aufsatz „die Überlieferung gegen moderne Anfechtungen verteidigt“ habe ${ }^{13}$.

Unvermittelt beendet indes von Stern in seinem Brief vom 24. Oktober seine Überlegungen zu Athenern, Spartanern und Persern - und lässt die Gegenwart hereinbrechen:

„Persönlich kann ich wenig tröstliches melden. Habe im Sommer einen Monat auf Berezan gegraben - vielfach gestört durch die Potemkinaffäre und die Odessaer Ereignisse, Habe dann einen weiteren Monat in Livland gearbeitet, mit dem Brauning (sic! für Browning) auf dem Tisch in offener Revolution; dann viel zu tun gehabt auf dem Kongress von Jekaterinoslaw“14.

Auf der Insel Berezan im Schwarzen Meer, nahe Olbia, erforschte von Stern seit 1903 eine bis ins 7. Jahrhundert v. Chr. zurückreichende Kolonie Milets ${ }^{15}$, wobei er die Meuterei auf dem Linienschiff Potemkin vom 27. Juni bis 8. Juli 1905 wie die Auseinandersetzungen in Odessa aus großer Nähe verfolgen konnte. Beides gehörte in den Kontext der revolutionären Unruhen, die nach den Niederlagen im Russisch-Japanischen Krieg seit Januar 1905 immer wieder ausbrachen. Diese Unruhen weiteten sich auch in die baltischen Provinzen des Reichs aus, wo sich zahlreiche Esten und Letten insbesondere gegen die deutschbaltischen Gutsbesitzer erhoben. Von daher erklärt es sich, dass von Stern sich im Sommer 1905 auch auf dem von einem Bruder bewirtschafteten Familiengut Seyershof in Livland bewaffnet aufhielt.

Der Brief berichtet weiter von der Wahl von Sterns zum Dekan „durch das Vertrauen meiner Collegen“. Dies Vertrauen gegenüber einem adligen Deutschbalten inmitten aller Wirren in Odessa ist sehr bemerkenswert. Von Stern muss eine große persönliche Autorität besessen haben, die ihn auch kurz nach dem Ersten Weltkrieg in schwieriger Zeit 1921-1922 und nochmals 1923-1924 zum Rektor der Universität Halle hat werden lassen.

Gleichwohl betrachtete von Stern die Situation voller Pessimismus und strebte fort. Die Universität sei zwar soeben nach zehnmonatlicher Pause wiedereröffnet worden,

„aber auf wie lange? Wir leben in voller Anarchie. Wenn es möglich wäre, den Staub von den Füssen zu schütteln [...] emigrieren - ich täte es mehr wie gern. Leider ist dazu keine Aussicht vorhanden“.

\section{III}

Dieser deutliche Hilferuf traf bei Meyer auf offene Ohren und er wusste auch Rat. Er war 1902 als Professor für Alte Geschichte an die Universität Berlin berufen worden, sein Nachfolger in Halle, Ulrich Wilcken (1862-1944), war soeben im Begriff nach Leipzig zu gehen. Über die Wiederbesetzung der Professur in Halle hatte Meyer schon mehrmals mit dem dort lehrenden Klassischen Philologen Georg Wissowa (1859-1931) korrespondiert; nunmehr wandte er sich in einem Brief vom 12. Dezember 1905 an seinen Freund:

${ }_{13}$ Meyer 1954, 454 n. 1; vgl. Meyer 1924, 522 n. 1, wo er das türkische Verhalten im Herbst 1914 als Analogie heranzieht. Meyer nennt auch nicht: Busolt, 1905, der sich eingehend kritisch mit von Stern auseinandersetzt. Übrigens finden - ohne Bezug auf die frühere Kontroverse - beide Positionen auch heute Verfechter, die von Sterns etwa bei Welwei 1999, 77, die Meyers bei Meier 1995, 291-295, immerhin mit der Bemerkung: „Der Hinweis auf eine weitere persische Invasion war vielleicht nicht ganz so abwegig, wie er erscheint" (292), freilich bezüglich der spartanischen Argumentation (Thuk. I 90).

14 Es geht um den 13. archäologischen Kongress in Jekaterinoslaw (15.-27. August 2005).

15 Stern 1909, zu Berezan: 142-144; Solovyov 1999, 20-22. 
„E.v. Stern schrieb mir neulich aus Odessa einen ganz verzweifelten Brief, was ja begreiflich genug ist, bei den wüsten Zuständen, die dort herrschen: er sehnt sich fort. Wäre es möglich, ihn bei einer Berufung mit zu nennen? Die entgegenstehenden Bedenken verhehle ich mir keineswegs; namentlich wird ihm ja alles nichtgriechische ziemlich fern liegen, aber ich möchte doch, daß Ihr (ich denke speciell an Dich und Robert) wenigstens einmal die Frage aufwürfet, ob man ihn mit nennen soll“16.

Das war recht vorsichtig formuliert, und in der Tat fand dieser Vorschlag in Halle zunächst wenig Gegenliebe. Am 29. Dezember 1905 berichtete Wissowa über die dortigen Überlegungen. Favorit für die Neubesetzung war Benedictus Niese (1849-1910), während die Nominierung von Sterns bei Wilcken und Carl Robert auf Widerstand stieß, ersterer auch mit dem Argument, wenn man schon nach Russland sehe, dann solle man „besser den entschieden bedeutenderen Rostowzew vorschlagen“. Schließlich bat Wissowa um genauere Orientierung über die Arbeiten von Sterns. Diesen Wunsch erfüllte Meyer umgehend am 30. Dezember 1905. Er würdigte dann aber nur kurz die Dorpater Dissertation und eine weitere Arbeit zu Xenophon ${ }^{17}$ als förderlich für die Forschung, um zu enden:

„Überhaupt hat, was er gemacht hat, immer Hand und Fuss, wenn ich auch nicht allem beistimme, und ist durchweg respectabel. Die gegen ihn vorliegenden Bedenken verkenne ich keineswegs. [...] Mein Hauptgrund über ihn an Dich zu schreiben, war also, daß ich doch wenigstens die Erwägung anzuregen wünschte, ob man ihn nicht aus der traurigen Situation in Odessa erlösen könne - ein solcher Grund liegt bei Rostowszew (sic) meines Wissens nicht vor“.

Besonders überschwänglich war diese Würdigung eben nicht. Bemerkenswert ist dabei, dass Meyer die von ihm besprochene Arbeit von Sterns zum Ephorat eben so wenig erwähnt hat wie dessen große Rezension zu seiner,Geschichte des Altertums' oder die Kontroverse um den Mauerbau des Themistokles. Dies alles verbirgt sich allenfalls hinter der lakonischen Bemerkung: „wenn ich auch nicht allem beistimme“.

Offenbar empfand Meyer nunmehr aber selbst das Bedürfnis, sich über das wissenschaftliche Werk von Sterns genauer zu informieren. Auf eine entsprechende Anfrage antwortete von Stern am 21. Januar 1906, dass er eben nur einige russisch geschriebene Aufsätze zur Verfügung habe. Zwei grössere deutsch und russisch verfasste Arbeiten „über die prähistorische griechische Cultur in Süd-Russland und über die Geschichte Theodosias“ 18 könnten „der ewigen Streiks und Revolutionen wegen“" seit langem nicht gedruckt werden.

Er geht dann zu einer Schilderung der Verhältnisse in Odessa über. Die Universität funktioniere seit einem Jahr nicht mehr und auch jetzt sei keine Aussicht auf einen Lehrbetrieb vorhanden. Dafür habe er als Dekan wegen fast permanenter Sitzungen des Conseils und als Glied und Präsident von zehn Kommissionen fast jeden Tag 5-6 Stunden Sitzungen mitzumachen oder zu leiten.

„Ein Ende ist noch gar nicht abzusehen und da noch täglich hier Attentate und Bombenwerfereien stattfinden, wie man unversehrt aus diesem Hexensabbath hervorgehen sollte“.

\footnotetext{
16 Audring 2000, 194. - Danach auch die folgenden Briefe zwischen Meyer und Wissowa.

17 Stern 1884; Stern 1887.

18 Stern 1906; die andere Arbeit ist offenbar so nicht im Druck erschienen.
} 
Dabei macht von Stern auch seinen eigenen Standort in dieser Situation klar:

„Wir schwanken zwischen roter Revolution und schwarzer Reaktion steuerlos einher und die gemässigten liberalen Elemente haben es besonders schwer, in diesem Kampfe zur Geltung zu kommen und nicht zerrieben zu werden. [...] Gelingt es bis zum Frühling die Duma unter Dach zu bringen, so kann vielleicht eine Besserung der Verhältnisse eintreten; ich setze auf sie keine allzu großen Hoffnungen“.

In Halle zog sich die Neubesetzung der Professur in die Länge. Nachdem Adolf Bauer (1855-1919) abgesagt hatte, kam es im Juli 1906 zu Berufungsverhandlungen mit von Stern im Berliner Ministerium. Diese führten freilich zunächst zu keinem Ergebnis und wurden erst Ende August wieder aufgenommen. Von Stern musste in seinem Brief vom 27. August 1906 an Meyer konstatieren, dass er nunmehr nicht vor dem Sommersemester 1907 kommen könne. Das Universitätsjahr habe soeben begonnen und er habe sich dem Minister gegenüber verpflichtet, das Präsidium in der Staatsexamenskommission zu übernehmen. Diese Verzögerung aber wollte man in Berlin nicht akzeptieren. Am 21. September teilte daraufhin Wissowa brieflich Meyer mit, dass jetzt Niese ernannt worden sei.

\section{IV}

Rückblickend beklagte sich von Stern am 8. Oktober 1906 nochmals bei Meyer über die Verzögerungstaktik des Personalreferenten für Universitätsangelegenheiten im Preußischen Kultusministerium, Ludwig Elster (1856-1935). Deshalb habe er zum Wintersemester nicht kommen können und daraufhin sei Niese vorgezogen worden. Offenbar hat er nunmehr die Situation in Odessa zwar als schwierig, aber doch nicht als so bedrohlich eingeschätzt, dass er um jeden Preis fortstrebte. Gegenüber Meyer schilderte er sie so:

„Hier ist eben alles in vollem Gang, so sehr, dass man nicht zum Atmen kommt. Vorlesungen auf der Doppeluniversität ${ }^{19}$, Examina in der Staats-Kommission und Universität, Conseil, Senat, Fakultät, eine Fülle Verwaltungssachen - und dabei ein Boden, der von der roten und schwarzen Revolution gleichmässig erhitzt wird. Für's Erste geht es, mir scheint, dass die Revolution etwas abflaut, wenigstens ist die Gesellschaft nüchterner als im Vorjahr, aber den Propheten spielen ist schwer, doppelt schwer im Land der ,Wosmoshnitäten' (sic!) (Möglichkeiten), wie Dernburg einmal Russland treffend genannt hat ${ }^{“ 20}$.

Bald darauf kehrte er zunächst noch mehr in die Normalität zurück. Er dankte am 4. Januar 1907 Meyer für einen ausführlichen Brief. Es sei wohltuend, „in der wissenschaftlichen Vereinsamung [...] Anregung und Förderung in den Fragen zu erhalten, die man hier allein mit sich abzumachen hat". So denke er oft an das Ovidische: barbarus hic ille sum, quia non intellegor ulli ${ }^{21}$. Material hätte er "hier in Fülle“, aber die administrativen Anforderungen seien hoch. In sieben verschiedenen Kommissionen sei er der Präsident. Dennoch hoffe er, „meine grosse Arbeit über die Milesische Kolonisation auf Grund der Ausgrabungen auf Beresan, das diese Kolonisation in ganz neuer Form giebt, zu Ende zu

${ }^{19}$ Neben der Universität war von Stern für die Höheren Frauenkurse sehr engagiert.

${ }^{20}$ Dernburg1885.

${ }^{21}$ Ovid, Tristia 5, 10, 37; der Text lautet genauer: barbarus hic ego sum, qui non intellegor ulli. 
bringen. Für den nächsten Juni hat die Regierung trotz Revolution wieder den nötigen Kredit zur Fortführung der Grabung bewilligt“.

„Was die Liste betrifft" - wahrscheinlich hatte Meyer gefragt, welche russischen Gelehrten zum Dritten Internationalen Historikerkongress nach Berlin im August 1908 eingeladen werden sollten und dabei schon einige Namen genannt, - so fügt von Stern für St. Petersburg hinzu Prof. Shebelev ${ }^{22}$ und „meinen Schüler" Pharmakowsky ${ }^{23}$. „Letzterer, Mitglied der Kaiserl. Archeol. Kommission, ist jedenfalls bedeutender als Pridik “24. Für Moskau nennt von Stern Prof. Al. Nikitsky ${ }^{25}$, „unseren ersten Epigraphiker, Dekan der hist. Fakultät, bekannt durch seine Arbeiten über Delphi“, für Charkow Prof. Vl. P. Buzeskul (Einleitung in die Geschichte Griechenlands, Perikles, die 'A $\theta \eta v . \pi 0 \lambda$.) ${ }^{26}$, für Kiew Prof. Jul. A. Kulakowsky ${ }^{27}$, für Kasan Prof. Serg. P. Shestakow ${ }^{28}$.

Dann aber folgt doch wieder ein düsteres Zeitbild:

„Wir leben hier im Übrigen wie in Wild-West mit dem Revolver auf dem Tisch; ,Expropriationsüberfälle giebt es noch täglich, aber man ist ziemlich abgestumpft dagegen und geht ruhig seinen Geschäften nach. Die Lage in der Universität ist nach wie vor precär; wie das nächste

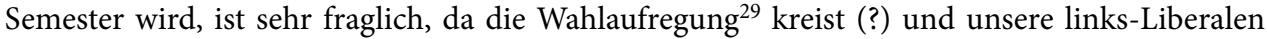
unverbesserlich sind; im Ganzen aber hat der Revolutionstaumel nachgelassen, zum Teil freilich nur unter dem Druck eines ähnlichen Taumels des reaktionären, Verbandes der russischen Leu$\mathrm{te}^{\mathrm{c}}{ }^{30}$. Ob und wann aus den beiden Extremen sich eine vernünftige Mittelpartei krystallisieren wird, die eine Realpolitik treibt, bleibt abzuwarten.

Im nächsten Brief vom 20. Januar 1907 zeigt sich der nämliche Zwiespalt. Zunächst dankt von Stern für die Zusendung von Meyers Abhandlung, Sumerier und Semiten ${ }^{31}$. Er müsse gestehen, „ich habe bisher die Rolle der Sumerier in vielen Punkten über-

22 Sergej A. Zhebelev (1867-1941), Epigraphiker und Althistoriker, seit 1904 extraord. Professor für Klassische Philologie an der Universität Sankt Petersburg, später ord. Professor und Akademiemitglied (s. Frolov 2006, 293-307 und zur Laufbahn Словарь профессоров и преподавателей Санкт-Петербургского университета 1819-1917 [Lexikon der Professoren und Dozenten der Univ. Sankt Petersburg]: http:// bioslovhist.history.spbu.ru/component/fabrik/details/1/861.html.

${ }^{23}$ Boris W. Pharmakowsky (1870-1928), Archäologe und Kunsthistoriker, in dieser Zeit PD an der Universität Sankt Petersburg, später ord. Professor und korr. Akademiemitglied, zu seiner Laufbahn s. Словарь профессоров и преподавателей Санкт-Петербургского университета 1819-1917 [Lexikon der Professoren und Dozenten der Universität Sankt Petersburg]: http://bioslovhist.history.spbu.ru/ component/ fabrik/details/1/612-farmakovskij.html. Stern gab später eine Würdigung seiner Arbeiten in: Stern 1915 [Штерн Н. А. Ученые заслуги Б. В. Фармаковского].

${ }^{24}$ Evgenij M.Pridik (1865-1935), Numismatiker und Epigraphiker, custos der Abteilung der Antiquitäten der Eremitage, Mitarbeiter von Latyschevs IOSPE, s. Pavlichenko 2000 [Павличенко Н. А. Е.М.Придик, петербургский филолог и эпиграфист].

${ }_{25}$ Alexander I. Nikitskij (1859-1921), Epigraphiker, ord. Prof. für Klassische Philologie an der Universität Moskau (1902-1910), später in Sankt Petersburg, korr. Akademiemitglied (1902, seit 1917 ord. Mitglied), s. Frolov 2006, 289-291.

${ }^{26}$ Wladislav P. Buzeskul (1858-1931), Althistoriker, ord. Prof. für allgemeine Geschichte an der Universität Charkow (1895-1924), Akademiemitglied (1922), s. Frolov 2006, 356-372.

${ }^{27}$ Julian A. Kulakowskij (1855-1919), Althistoriker, Byzantinist und Archäologe, ord. Professor für klassische Philologie an der Universität Kiew (1884-1919), s. Frolov 2006, 342-349.

${ }^{28}$ Sergej P. Shestakow (1864-1940), Altphilologe und Byzantinist, ord. Prof. für Klassische Philologie an der Universität Kazan (seit 1902), korr. Akademiemitglied (1916), s. Frolov 2006, 339.

${ }^{29}$ Wohl die Wahlen zur 2. Duma (Februar-Juni 1907).

${ }^{30}$ Der orthodox-monarchistische „Bund des russischen Volkes“ hatte sich November-Dezember 1905 gebildet.

31 Meyer 1906. 
schätzt und bin erst durch Ihre Darlegungen zur Einsicht gelangt, dass ich den Semiten in manchem Unrecht getan habe" worauf er im Detail doch Zweifel an der kulturellen Einwirkung der Semiten auf die Sumerier geltend macht. Unvermittelt folgt aber die Gegenwart in Odessa:

„Aus den Zeitungen wissen Sie wohl, dass bei uns noch täglich flott geraubt und gemordet wird, auch Bomben sind an der Tagesordnung. Neulich geriet ich zweimal im Lauf eines Tages in einen recht regen Kugelwechsel hinein; jedesmal bis 50 Schuss, wunderbarer Weise sind wenig aus dem Publikum verletzt. Die Arbeit geht dabei ihren Gang; Vorlesungen, Sitzungen — der Tod jeder wissenschaftlichen Tätigkeit - nehmen den ganzen Tag und Abend in Anspruch [...] dazu Examina ohne Ende [...]“.

Der nächste Brief datiert erst vom 27. Oktober 1908. Inzwischen hatte von Stern am Internationalen Historikerkongress in Berlin teilgenommen und am 7. August 1908 einen Vortrag vor den vereinten Sektionen für Geschichte von Hellas und Rom und für Archäologie, wesentlich auch zu eigenen Forschungen am Schwarzen Meer, gehalten ${ }^{32}$. Erneut dankte er Meyer für seine „liebenswürdige Gastfreundschaft“ und entschuldigte seine überstürzte Abreise auf die Nachricht vom Tode seines Bruders Robert (1855-26.7.1908) hin. Es sei die Leitung des Tschernigower Kongresses gefolgt ${ }^{33}$ und die Neuaufstellung des Museums in Odessa, dessen Direktor von Stern seit 1895 erfolgreich gewesen ist.

Unvermittelt aber berichtet er dann

„von einer schamlosen Hetze in der chauvinistischen Presse, verbunden mit Strassenüberfällen und Drohungen und Unannehmlichkeiten aller Art, und dies Alles, weil die Mehrzahl meiner Kollegen mich zum Rektor wählen wollte; den ,wahrhaften Russen' ist der Deutsche und Nichtortodoxe (sic!) auf diesem Posten ein Dorn im Auge. Ich habe um alle Weiterungen zu vermeiden, meine Kandidatur zurückgezogen, trotzdem die grosse Mehrzahl der Studenten mich bat es nicht zu tun und wir haben nun einen Rector nach dem Herzen der nationalen Machthaber, vor allem unseres Herrn über Leben und Tod, des beim Kriegszustand allmächtigen Generalgouverneurs".

Bei allen Problemen wird doch auch das große Ansehen deutlich, das von Stern in Odessa zuteil geworden ist; das bezeugt ebenso die Fortsetzung:

„Nachher bin ich dann freilich zum Direktor des weiblichen Höheren Frauenkurses erwählt, den c. 1400 Zuhörerinnen haben [...] — da dies ein Privatinstitut ist, schweigt der Chauvinismus fürs Erste. Die Zustände werden allmählich recht unleidlich; erst ging der Pendel erschreckend nach links, jetzt ebenso erschreckend in die andere Richtung, bis die aristotelische

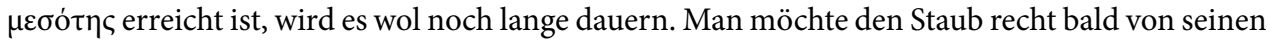
Füssen schütteln. Die einzige Freude ist mein Museum — das ist wirklich ansehnlich geworden“.

Bald darauf, am 6. Februar 1909, dankte von Stern für die zweite Hälfte des ersten Bandes der ,Geschichte des Altertums' in der zweiten Auflage. In einem Punkte meldete er sogleich Widerspruch an. Die prähistorischen Kulturen im Schwarzmeergebiet seien

32 Stern 1909; vgl. auch Stern 1915.

33 Archäologischer Kongress in Tschernigow 1.-12. August 1908. 
keineswegs von der Ägäis her beeinflusst worden ${ }^{34}$, ganz im Gegenteil habe man „an eine Kulturwelle von Norden nach Süden in der neolithischen Zeit zu glauben“.

In einem weiteren Brief vom 30. Mai 1909 empfahl von Stern den Privatdocenten E. G. Kagaroff (1882-1942), der mit einem zweijährigen Reisestipendium „in Berlin die Lücken seiner philol. Ausbildung füllen und seine religionsgeschichtlichen Untersuchungen weiter führen“ solle ${ }^{35}$. Anschließend berichtete er kurz von einer Ägyptenreise, auf der er auch den Ägyptologen Georg Steindorff (1861-1951) getroffen habe. „Der Congress selbst (sei) freilich recht schwach (gewesen) - zum Teil fast eine Parodie auf die Wissenschaft" 36 .

Am 31. August 1909 dankte von Stern u. a. für die Zusendung von Meyers Schrift über Theopomp ${ }^{37}$. Er stimmte Meyer darin zu, dass Theopomp als Verfasser der Hellenika von Oxyrynchus anzusehen sei, widersprach aber dessen Ansicht, dass die Schlacht von Sardes bei Theopomp richtiger geschildert werde als bei Xenophon. In die Gegenwart übergehend schrieb er dann:

„Sie gehen auf längere Zeit nach Amerika ${ }^{38}$ - ich beneide Sie darum, ich möchte auch gehen, wenn nicht nach Amerika, so doch fort von hier; die nationalen Gegensätze spitzen sich derart zu, wie ich das vor zwei Jahren noch für undenkbar gehalten hätte - wenn man heutzutage nicht Vollblutrusse und Orthodoxer ist, der in das chauvinistische Horn tutet, dem wird der Boden für die Existenz abgegraben. [...] Sie können sich nicht vorstellen, wie unerquicklich die Verhältnisse sind, wie man auf Schritt und Tritt in der ultranationalen Presse beschimpft und verhöhnt wird und wie ganz machtlos man all diesem Treiben gegenüber ist. Dabei geht Arbeitsmut und die Lust am Weiterwirken einfach zum Teufel. Doch genug der Jeremiade [...]“.

Nun aber traf von Stern ein besonders schwerer Schicksalsschlag: der Tod seines jüngeren Sohnes Arist Rudolf (1894-25. März 1910) nach kurzer, schwerer Krankheit. Am 20. April 1910 dankte von Stern Meyer für dessen „warme Teilnahme“ und schilderte seinen hochbegabten und allseits geliebten Sohn, der bereits zu einem wichtigen Mitarbeiter für ihn geworden sei.

Dann wendet er sich der Vakanz der Professur in Halle nach dem Tode von Niese am 1. Februar $1910 \mathrm{zu}$. In der Tat hatte, wie Wissowa schon am 6. März 1910 Meyer mitteilte, die Fakultät rasch gehandelt und auf ihrer Liste nach Conrad Cichorius (1863-1932) von Stern an 2. Stelle genannt. Der Personalreferent im Ministerium, Elster, wolle aber erst die Rückkehr Meyers aus den USA abwarten, um mit ihm diese Frage $\mathrm{zu}$ besprechen. Eben dies hatte auch von Stern, wie er seinerseits Meyer schrieb, von seinem Landsmann in Berlin, dem Professor für Osteuropäische Geschichte Theodor Schiemann (1847-1921) erfahren. Er wende sich jetzt aber an Meyer, um ihm offen mitzuteilen, dass er sich

„in einer seelischen Depression (befinde), die derart ist, dass ich mich einem neuen Wirkungskreis gegenüber nicht gewachsen fühle. Ich leiste mechanisch das grosse Arbeitspensum, das das eiserne Muss des Tages mir auferlegt, aber für Halle wäre das zu wenig. [...] Ich

${ }^{34}$ So noch Meyer 1913, 825; dagegen auch Stern 1909, 140.

35 Zu Kagaroff: Frolov 2006, 341.

${ }^{36}$ Freundlicher berichtet Toutain 1909. Von Sterns Referat: Stern 1909.

37 Meyer 1909.

${ }_{38}$ Meyer ging im Rahmen des amerikanisch-deutschen Austauschprogramms im Wintersemester 1909/10 an die Harvard University und dann noch weiter auf Reisen. 
hielt es für meine Pflicht Ihnen ganz ehrlich meinen Zustand zu schildern, da Ihre Stimme in der Frage von Ausschlag gebender Bedeutung ist und Sie daher vollkommen orientiert sein müssen“.

\section{VI}

Eine Woche später, am 28. April 1910, hatte von Stern zunächst eine ganz andere Anfrage: „Ein Student [...] hat den ersten Teil des I. Bandes Ihrer Geschichte des Altertums ins Russische übersetzt und brachte mir vor ein paar Tagen das Manuskript zur Durchsicht“. Natürlich werde er das nur mit Meyers Einverständnis übernehmen, allerdings gebe es keine Literaturconvention zum Schutze des geistigen Eigentums zwischen Russland und dem Westen. Beloch sei in ähnlicher Lage gewesen und habe sogar „einen Brief als Einleitung zur Übersetzung (geschrieben), da er sie doch nicht hindern konnte. Ich habe dem Studenten - er ist natürlich ein Jude - den Standpunkt klar gemacht, dass es der literarische Anstand erfordere, Ihre Einwilligung einzuholen, und er giebt zu, das begriffen zu haben und hat mich auch gebeten, die Vermittlung zu übernehmen“. Freilich sei keineswegs sicher, was bei fehlender Einwilligung geschehen werde. „Ich kenne den Herrn dazu viel zu wenig, und dann ist er ein Jude, der als Philolog und Historiker keine andere Verwertung seiner Kenntnisse hat, als die Umsetzung in Druckerschwärze“.

Daran fügt aber von Stern nochmals Bemerkungen in eigener Sache an:

„Mein Arzt, dem ich Mitteilung gemacht habe vom Inhalt des letzten Briefes [...] ist sehr ungehalten über denselben. Er meint, ein Orts- und Tätigkeitswechsel sei das beste, fast einzige Mittel gegen die seelische Depression, an der ich leide. Möglich, dass er Recht hat - aber ich hielt es doch für meine Pflicht, Sie über den desolaten Zustand aufzuklären, in dem ich mich eben befinde. Wie Sie sich zu dieser Aufklärung stellen, ist ja Ihre Sache. Ich habe im letzten Jahr nur ein paar russisch geschriebene Arbeiten publiciert: ein deutscher Aufsatz über hellenistische Keramik im Zusammenhang der Handelsbeziehungen in hellenistischer Zeit zwischen Alexandrien und dem Pontosgebiet wird etwa in 14 Tagen erscheinen“39.

Das war eine zwar vorsichtig formulierte, aber doch deutliche Interessenbekundung an einem Ruf nach Halle. Der nächste Brief von Sterns vom 6. Mai 1910 lässt nichts von Meyers Reaktion darauf erkennen. Es geht nur um die unautorisierte Übersetzung ins Russische, die Meyer offenbar wenig erfreut hat. Von Stern bemerkt dazu:

„Natürlich haben Sie Recht, dass der Übersetzer sich vorher an Sie hätte wenden müssen; hätte er mir seine Absicht mitgeteilt, ehe er mit dem Manuscript anrückte, so hätte ich ihn natürlich an Sie gewiesen; von einem skytisierten Juden ist besonderer Takt freilich nicht zu erwarten. Den russischen Schriftstellern - um die Gelehrten oder Quasi-Gelehrten kümmert sich die Welt im Ganzen wenig - ergeht es übrigens nicht besser, wie Ihnen. Sie werden jenseits der Grenzpfähle auch verdeutscht, ohne gefragt zu werden. Solamen tamen est, socios habuisse malorum ${ }^{40}$. Spielhagen $^{41}$ war meines Wissens so gewitzigt, dass er, um sich vor Freibeuterei zu schützen, seine Werke gleichzeitig in Riga verlegte. Das müssen Sie in Zukunft Cotta (sc. Meyers Verleger)

39 Stern 2010 [К вопросу об эллинистической керамике]; von Stern erwähnt nicht seine Aufsätze von 1909.

40 In der Form: Solamen miseris socios habuisse malorum nach Äsop ,Die Hasen und die Frösche u. a. bei Spinoza.

${ }^{41}$ Friedrich Spielhagen (1829-1911), ein damals viel gelesener deutscher Schriftsteller. 
auch anraten. Wenn ich den Übersetzer zu Gesicht bekomme [...] werde ich ihn an Cotta weisen, sowohl wegen der Neuauflage als auch um die Sache überhaupt in Ordnung zu bringen. Ist das geschehen, so will ich im Sommer die Arbeit durchsehen“.

Einen Monat später aber wird aus dem Brief vom 6. Juni 1910 deutlich, dass von Stern jetzt auf den Beginn der Berufungsverhandlungen mit Elster warten durfte. Offensichtlich hat sich also Meyer für ihn beim Ministerium erfolgreich eingesetzt und ihm das sogleich mitgeteilt. Allerdings verging doch noch ein halbes Jahr, bis Wissowa am 26. Dezember 1910 Meyer schreiben konnte:

„Eben erhalte ich ein Telegramm von E.von Stern: ,Nehme zu Ostern Ruf an.' So ist denn diese endlos lange Berufungsgeschichte glücklich am Ende angelangt“.

\section{VII}

Von Sterns Ablösung von seinem Wirkungskreis in Odessa war, wie wir gesehen haben, langwierig - und wohl noch schmerzlicher, als die Briefe an Meyer ahnen lassen. Er war im Leben der dortigen Universität bestens integriert, wirkte als Dekan und beinahe noch als Rektor. Wissenschaftlich hatte er eine erfolgreiche Grabungstätigkeit und den Aufbau eines bedeutenden Museums vorzuweisen, das er von 1895-1910 als Direktor geleitet hat. Er war eine Autorität für die Geschichte des nördlichen Schwarzmeergebietes. Zudem hatte er auch zahlreiche akademische Schüler, insbesondere Michael Mandes. An Anerkennungen dafür fehlte es nicht. 1907 wurde er Mitglied der Kaiserlichen Archäologischen Kommission in St. Petersburg und 1906 zum Kaiserl. Russischen Wirklichen Staatsrat ernannt; er erhielt mehrere hohe Orden; 1911 wurde er in die Matrikel des erblichen russischen Adels im Heraldie-Departement des Senates aufgenommen.

Sein Abschied von Odessa vollzog sich in allen Ehren. Der Rat der Odessaer Gesellschaft für Geschichte und Altertümer beschloss am 5. März 1911:

„1. E. R.von Stern zu bitten, auch in der Zeit seines Aufenthaltes außerhalb von Odessa Mitglied des Rates der Gesellschaft zu bleiben; 2. Im Sitzungssaal der Gesellschaft ein Porträt E. R. von Sterns anzubringen; 3. Den 30. Band der Mitteilungen der Gesellschaft E. R. von Stern zu widmen und 4. E. R. von Stern im Namen der Gesellschaft eine Grußadresse zu schicken “42.

Die Festschrift ist in Odessa im Jahre 1912 erschienen. Sein Porträt befindet sich noch heute in Odessa ${ }^{43}$, wo sein Andenken gemäß der homepage des Museums immer noch hochgehalten wird. Auf der Insel Berezan hat er im Jahre 1913 nochmals Grabungen durchgeführt. Auch durch seinen älteren Sohn Viktor Axel, der an der Universität Dorpat / Jurjew Jura studierte und einmal das Familiengut Seyershof in Livland übernehmen sollte, blieb er mit Russland verbunden.

Ohne die Revolution von 1905 und die folgende Aufheizung des politischen Klimas wäre ein derart gut integrierter Gelehrter kaum auf den Gedanken gekommen, nach Deutschland zu wechseln. Insofern ist sein persönlicher Entschluss zugleich ein Beleg dafür, in welchem Ausmaß Revolution und Reaktion die übernationale Reichselite geschwächt haben, die das russische Imperium jahrhundertelang erfolgreich geführt hat.

${ }^{42}$ Häusler 1984, 686; die Urkunde im Facsimile, 687-692.

43 Vgl. die Abbildung in Ungern-Sternberg 2015. 
Mehrfach wird in den Briefen deutlich, wie von Stern vergeblich nach einer liberalen Mittelposition Ausschau gehalten hat, von der aus eine fruchtbare Reformpolitik hätte betrieben werden können.

\section{VIII}

Mit von Sterns Wechsel nach Halle bricht der Briefwechsel mit Meyer ab. Möglicherweise haben sie sich indes persönlich in Halle, wohin Meyer viele Beziehungen hatte, oder auch in Berlin getroffen. Während des Ersten Weltkriegs engagierten sich beide publizistisch - Meyer mit dem Fokus auf die angelsächsische Welt ${ }^{44}$, von Stern sehr viel zurückhaltender mit einigen Broschüren zu den russischen Verhältnissen, die seine tiefe Vertrautheit mit diesen dokumentieren ${ }^{45}$.

So war es auch eine Anfrage Meyers an den Russlandexperten von Stern, die dessen letzten erhaltenen Brief an Meyer vom 25. Januar 1918 zur Folge hatte. Sie betraf die heftige Fehde, die zwischen dem deutschbaltischen Historiker Johannes Haller (1865-1947) und dem Historiker und Osteuropaexperten Otto Hoetzsch (1876-1946) im Gange war. Hoetzsch war in der ,Kreuzzeitung für eine Verständigung mit Russland und gegen Annexionen eingetreten, weshalb ihn Haller in einer Broschüre „Die russische Gefahr im deutschen Hause" (Stuttgart 1917) heftig und auch persönlich beleidigend angegriffen hatte. Hoetzsch antwortete darauf mit: „Russische Probleme. Eine Entgegnung auf J. Hallers Schrift ,Die russische Gefahr im deutschen Hause "“ (Berlin 1917), in der er seinerseits Haller ,jede wissenschaftliche Legitimation in Rußlandfragen absprach“ ${ }^{46}$.

„Hallers Tonart" wollte von Stern nicht überall billigen, in der Sache freilich gab er ihm überwiegend Recht. Hoetsch's (sic!) Schriften bewiesen, „dass er über russische Verhältnisse keine auf Autopsie beruhende Anschauung" habe. Sein Urteil sei

„durch die Überlegung bedingt [...], wir müssten auf alle Fälle suchen, mit unserem östlichen Nachbar gute Freundschaft zu halten. Dass dieser Versuch, der ja oft gemacht ist, bisher keinen Erfolg gehabt hat und auch nicht haben kann, wenn wir nicht Oestreich und die Türkei opfern und uns wie zu Zeiten Nikolaus I. unsere Politik von Russland vorschreiben lassen wollen, ficht Prof. Hoetsch nicht an“.

Zur „Farce von Brest-Litowsk“, wo die Verhandlungen seit dem 18. Januar 1918 unterbrochen waren, bemerkt von Stern anschließend: „Wichtig wäre es, wenn es gelänge, mit der Ukraine zu einem Abschluss zu kommen; seit in Charkow die Maximalisten ${ }^{47}$ herrschen, ist aber auch das zweifelhaft ${ }^{\star 48}$.Trotzki andererseits, stellt er richtig fest,

„will offenbar gar keinen Frieden schliessen, sondern die Sache nur hinziehen; er hofft, auch wir bekommen Revolution und er könne dann mit dem ,edlen Held Liebknecht', - wie er ihn nennt, in Stockholm verhandeln, andererseits weiss er, dass sobald der Friede abgeschlossen ist, die Tage der Maximalisten gezählt sind — nur das grosse Friedensbedürfnis stärkt ihrer Gewaltherrschaft den Rücken. Ihm kommt es nur auf den Waffenstillstand an, um in dessen Schutz die

44 Sösemann 1990; Ungern-Sternberg 1990; Hoffmann 1991.

45 Stern 1918 (bis).

46 Mann 1965, 77.

47 Eine zeitgenössische Bezeichnung der Bolschewisten.

48 Der Friede mit der Volksrepublik Ukraine wurde am 9. Februar 1918 geschlossen. 
maximalistische Agitation in den Grenzgebieten für das unsinnige Selbstbestimmungsrecht mit Volldampf zu betreiben - mit welchem Erfolg lehren leider die Vorkommnisse in den von uns unbesetzten Teilen Livlands und Estlands“.

Nun aber wendet sich von Stern Trotski mit persönlichen Erinnerungen zu:

„Unsere Diplomaten kennen leider den Ton nicht, in dem mit diesem Judenjüngling geredet werden muss. Seinem Vater, dem Juden Braunstein (sic!) aus Nikolajew, der mir mal gefälschte Altertümer anschmieren wollte, und als ich sie zurückwies, behauptete, ich verstände von der Sache nichts, habe ich ein Buch an den Kopf und ihn zur Türe hinaus geworfen und fand ihn dann nachher sehr entgegenkommend und traitabel — beim Sohn würden wohl drastische Mittel auch wirken, sind aber nicht anwendbar, wie die Dinge nun einmal liegen“.

Von Stern war in der Tat ein intimer Kenner der vor dem Ersten Weltkrieg blühenden Fälschungsindustrie von Antiquitäten in Südrussland und hat über diese - fast durchweg in russischer Sprache - wiederholt berichtet ${ }^{49}$. Es ist durchaus glaubhaft, dass er in seiner Eigenschaft als Museumsdirektor dabei auch dem Vater Trotzkis begegnet ist. Falls dieser, wie Trotzki in seiner Autobiographie behauptet, im Jahre 1879 ein Landgut gekauft hatte, so kann er doch nebenbei auch weiterhin als Händler tätig gewesen sein.

Anzufügen ist dabei, dass von Stern zwar in diesem privaten Brief an Meyer Trotzki so prononciert als Juden gekennzeichnet hat, in seinem Aufsatz ,Der Bolschewismus in Theorie und Praxis' aber im Jahr 1919 von Juden nirgends die Rede ist. Einleitend behandelt er die Frage, inwieweit es sich bei dem Bolschewismus um ein internationalesmarxistisches Phänomen handele und kommt zu dem Ergebnis:

„Da aber die Vertreter dieser Theorien, die sie in die Praxis umzusetzen berufen waren, Russen sind - denn auch Trotzki-Bronstein, Kamenew-Rosenfeld, Radek-Sobelsohn sind trotz ihrer nichtrussischen Abstammung in russischem Ideenkreis aufgewachsen, sind in ihren Weltanschauungen und Bestrebungen Russen, sind es auch in ihrem eigenartigen Internationalismus - so erklärt es sich daß der Bolschewismus doch auch eine ganze Reihe spezifisch russischer Züge trägt“.

Wenn von Stern in seinem Brief an Meyer schließlich feststellt: „Das einzig angebrachte wäre seiner Verschleppungstaktik gegenüber, den Waffenstillstand zu kündigen und bis zur Peipusgrenze vorzurücken und somit das Fenster, das Peter durch die Einverleibung des alten Ordenslandes nach Europa geschlagen, wieder zu schliessen, nur dann haben wir im Osten eine dauernde Sicherung", so nimmt er damit den Vormarsch der deutschen Armee ab dem 17. Februar 1918 vorweg.

\section{IX}

Meyer und von Stern sind wohl nie enge Freunde geworden. Bezeichnenderweise blieb es bei der Anrede „Sehr geehrter Herr College“, oder auch „(Sehr) verehrter Herr College“, nur zwischen 1906 und 1908 und 1910 „Lieber Herr College“, um noch 1910 und

49 Несколько заметок по поводу статьи В.В.Латышева «О поддельных греческих надписях из южной России» [Stern 1895]; «О подделке предметов классической древности на юге России» [Stern 1896]; «О новейших подделках в области керамики на юге России» [Stern 1897, Über die neuesten Fälschungen; Stern 1897, Die Tiara des Saitaphernes]; «О новом способе подделки античных росписных ваз» [Stern 1906, Ein neues Verfahren]; «О новом способе фальсификации в серебре и мраморе» [Stern 1906, Über ein neues Verfahren]; Stern 1910; 1911. 
dann $1918 \mathrm{zu}$ „(Sehr) verehrter Herr College“ zurückzukehren. Auch die beiden bezeugten Besuche hatten jeweils einen besonderen Anlass, das Universitätsjubiläum in Halle und der Internationale Historikerkongress in Berlin. Wissenschaftlich hat von Stern die Werke Meyers achtungsvoll rezipiert, sich dabei aber durchaus sein eigenes Urteil bewahrend. Meyer seinerseits hat von den eigentlichen Forschungsleistungen von Sterns, der Archäologie und Geschichte der nördlichen Schwarzmeerküste, offenbar wenig Kenntnis genommen. Leider wissen wir nicht, wie er auf den letzten großen Aufsatz von Sterns zu den Gracchen reagierte ${ }^{50}$, den dieser ihm gewiss zugesandt hat. In ihm wird seinerseits die grundlegende Arbeit Meyers (n. 4) natürlich stets herangezogen. Dieser Aufsatz verarbeitet in einer sehr aufschlussreichen Weise nochmals die Eindrücke der russischen Revolutionen von 1905 und 1917 und den Umsturz in Deutschland 1918/19.

In einem entscheidenden Moment hat freilich Meyer auf das Lebensschicksal von Sterns bestimmend eingewirkt. Unter dem Eindruck der bedrohlichen Lage in Odessa während der Revolution von 1905 hat er von Stern für die Besetzung der althistorischen Professur an der Universität Halle ins Spiel gebracht und sein Votum dürfte auch den Ausschlag dafür gegeben haben, dass er im Jahre 1910 den Ruf nochmals erhielt. Für von Stern war dies im Hinblick auf den Ersten Weltkrieg und die folgende Revolution gewiss rettend, für die Universität Halle mit seinem zweimaligen Rektorat ein Gewinn.

\section{Bibliographie}

Audring, G. (Hg.) Gelehrtenalltag. Der Briefwechsel zwischen Eduard Meyer und Georg Wissowa (18901927). Hildesheim, Weidmann, 2000.

Audring, G. (Hg.) Eduard Meyer. Geschichte der ägyptischen und der babylonisch-assyrischen Literatur (1879). Speyer, Kartoffeldruck Verlag, 2009.

Beloch, J. Griechische Geschichte I. Straßburg, K. J. Trübner, 1893.

Beyschlag, W. Das zweihundertjährige Jubiläum der Universität Halle-Wittenberg. Halle, 1895.

Busolt, G. Thukydides und der themistokleische Mauerbau. Ein Beitrag zur Sachkritik, Klio 1905, 5, 255-279.

Calder III, W. M., Demandt, A. (Hg.), Eduard Meyer. Leben und Leistung eines Universalhistorikers. Leiden, E. J. Brill, 1990.

Dernburg, F. Russische Leute. Berlin, 1885.

Eduard Meyer (1855-1930). Zu Werk und Zeit. Wissenschaftliche Zeitschrift der Humboldt-Universität zu Berlin. R. Geistes- und Sozialwissenschaften 40, 1991.

Frolov, E. Eduard Meyer und die russische (bzw. sowjetische) Altertumswissenschaft, in: Eduard Meyer (1855-1930), 1991, 71-75.

Frolov, E. Russkaia nauka ob antichnosti: istoriograficheskie ocherki [Russische Altertumswissenschaft: historiographische Skizzen] (Sankt Petersburg, 2006²).

Häusler, A. Ernst von Stern, Archäologe in Odessa und Halle. Zum 125. Geburtstag. Ethnographisch-archäologische Zeitschrift 1984, 25, 683-695.

Hoffmann, Chr. Die Selbsterziehung des Historikers. Zur intellektuellen Entwicklung des jungen Eduard Meyer (1855-1879), in: Calder III - Demandt, 1990, 208-254.

Hoffmann, Chr. Eduard Meyers England- und Amerikabild, in: Eduard Meyer (1855-1930), 1991, 45-53.

Karo, G. Ernst von Stern. Biographisches Jahrbuch für Altertumskunde 1925, 45, 86-103.

Keil, B. Anonymus Argentinensis. Fragmente zur Geschichte des perikleischen Athen aus einem Strassburger Papyrus. Strassburg, K. J. Trübner, 1902.

Mandes, M. Messenskie voiny: istoriia i traditsiia. Messenskie voiny i vosstanovlenie Messenii [Die messenischen Kriege und die Wiederherstellung Messeniens. Geschichte und Tradition]. Odessa, 1898. (In Russian)

Mandes, M. Opyt istoriko-kriticheskogo kommentariia k grecheskoi istorii Diodora [Versuch eines historischkritischen Kommentars zur griechischen Geschichte Diodors]. Odessa, 1901. (In Russian)

50 Stern, 1921; dazu Ungern-Sternberg, 2015. 
Mann, B. Die baltischen Länder in der deutschen Kriegszielpublizistik 1914-1918. Tübingen, Mohr, 1965.

Maurer, T. Der Weg zur Mündigkeit. Auslandsaufenthalte rußländischer Wissenschaftler im 19. und frühen 20. Jahrhundert. Hyperboreus 2004,10, 60-77.

Meier, Chr. Athen. Ein Neubeginn der Weltgeschichte. München, 1995

Meyer, Ed. Rez. zu Stern 1894. Literarisches Centralblatt 1894, 1133.

Meyer, Ed. Geschichte des Altertums. III., IV. und V. Bd. Stuttgart, Cotta, 1901-1902.

Meyer, Ed. Der Mauerbau des Themistokles. Hermes 1905, 40, 561-569.

Meyer, Ed. Sumerier und Semiten in Babylonien. Abhandlungen Berliner Akademie, Nr. 3, 1906.

Meyer, Ed. Theopomps Hellenika. Mit einer Beilage über die Rede an die Larisäer und die Verfassung Thessaliens. Halle, Niemeyer, 1909.

Meyer, Ed. Geschichte des Altertums I 2. 3. Aufl. Stuttgart - Berlin, Cotta, 1913.

Meyer, Ed. Vorläufer des Weltkriegs im Altertum (1918), in: Kleine Schriften II, Halle, Niemeyer, 1924, 507-538.

Meyer, Ed. Geschichte des Altertums IV 1. 5. Aufl., Darmstadt, Wiss. Buchgesellschaft, 1954.

Pavlichenko, N. A. E. M. Pridik, Peterburgskii filolog i epigrafist [E.M. Pridik, Petersburger Philologe und Epigraphiker], in: Drevnii Mir i My 2, St.Petersburg, 2000, 189-206. (In Russian)

Sösemann, B. „Der kühnste Entschluss führt am sichersten zum Ziel“. Eduard Meyer und die Politik, in: Calder III - Demandt, 1990, 446-483.

Solovyov, S. L. Ancient Berezan. The architecture, history and culture of the first Greek colony in the Northern Black Sea. Leiden, Brill, 1999.

Stern, E. von. Geschichte der spartanischen und thebanischen Hegemonie vom Königsfrieden bis zur Schlacht bei Mantineia, Diss. Dorpat, H. Laakmann, 1884.

Stern, E. von. Xenophons Hellenika und die böotische Geschichtsüberlieferung. Eine historische Quellenstudie. Dorpat, Karow, 1887.

Stern, E. von. Zur Entstehung und ursprünglichen Bedeutung des Ephorats in Sparta. Berlin, Calvary, 1894.

Stern, E. von. Neskol'ko zametok po povodu stat'i V.V.Latysheva ,O poddelnych grecheskikh nadpisiakh iz iuzhnoi Rossii' [Einige Bemerkungen anlässlich des Aufsatzes von V.V.Latyshev über gefälschte griechische Inschriften aus Südrussland]. Zapiski Imp. Odesskogo obshchestva istorii i drevnostei 1895, 18, 65-86. (in Russian)

Stern, E. von. O poddelke pamiantnikov klassicheskoi drevnosti na juge Rossii [Über Fälschung von Gegenständen des klassischen Altertums in Südrussland]. Zhurnal Ministerstva Narodnogo Prosveshcheniia, Dez. 1896, 129-159. (In Russian)

Stern, E. von. O noveishikh poddelkakh v oblasti keramiki na iuge Rossii [Über die neuesten Fälschungen aus dem Gebiete der Keramik in Südrussland], Zapiski Imp. Odesskogo obshchestva istorii i drevnostei, 1897, 20, 37-41. (In Russian)

Stern, E. von. Die Tiara des Saitaphernes und die Goldfälschungen in Südrussland. Berliner philologische Wochenschrift 1897, 17, 764-768.

Stern, E. von. Rez. zu Mandes, 1898. Zapiski Imp. novoruss. univers., chast' 76, 1899, 61-70 (russ.).

Stern, E. von. Rez. zu Mandes, 1901. Zapiski Imp. novoruss. univers., chast' 87, 1902, 1-16 (russ.).

Stern, E. von. Rez. zu E. Meyer, 1901-1902, in: Göttingische Gelehrte Anzeigen 1903,165, 306-352.

Stern, E. von. Der Mauerbau in Athen und die List des Themistokles. Hermes 1904, 39, 543-562.

Stern, E. von. O novom sposobe poddelki antichnykh rospisnykh vaz [Ein neues Verfahren der Fälschung antiker gemalten Vasen]. Zapiski Imp. Odesskogo obshchestva istorii i drevnostei 1906, 26, V.5-15. (In Russian)

Stern, E. von. O novom sposobe falsifikatsii v serebre i mramore [Über ein neues Verfahren der Fälschung in Marmor und Silber]. Zapiski Imp. Odesskogo obshchestva istorii i drevnostei 1906, 26, VI.55-59. (In Russian)

Stern, E. von. Theodosia und seine Keramik. Odessa - Frankfurt a. M., J. Baer, 1906.

Stern, E. von. Die griechische Kolonisation am Nordgestade des Schwarzen Meeres im Lichte archäologischer Forschung. Klio 1909, 9, 139-152.

Stern, E. von. Relations commerciales entre l'Eypte et les colonies grecques situées sur le bord septentrional de la Mer Noire, in: Comptes rendus du deuxième congrès international d'archéologie classique. Alexandrie - Le Caire (7 avril - 15 avril 1909), II, Kairo, 1909, 225-229.

Stern, E. von. K voprosu ob ellinisticheskoi keramike. Ein Beitrag zur hellenistischen Keramik. Zapiski Imp. Odesskogo obshchestva istorii i drevnostei 1910, 28, 158-175; 176-190 (russisch und deutsch). 
Stern, E. von. Über Antikenfälschungen im Südlichen Russland. Mitteilungen des Museumsverbandes, Juli 1910, Nr. 256.

Stern, E. von. Über die neuesten Altertumsfälschungen. Mitteilungen des Museumsverbandes. Juli 1911, Nr. 310.

Stern, E. von. Die politische und sociale Struktur der Griechencolonien am Nordufer des Schwarzmeergebietes. Hermes 1915, 50, 161-224.

Stern, E. von. Uchenye zaslugi B.W.Farmakovskogo [Die wissenschaftliche Leistung von B.W.Pharmakowsky]. Moskau 1915.

Stern, E. von. Regierung und Regierte. Politiker und Parteien im heutigen Russland. Halle, 1918.

Stern, E. von. Die russische Agrarfrage und die russische Revolution. Halle 1918.

Stern, E. von. Der Bolschewismus in Theorie und Praxis. Hallische Universitätszeitung 1919, 1, 82-90.

Stern, E. von. Zur Beurteilung der politischen Wirksamkeit des Tiberius und Gaius Gracchus. Hermes 1921, $56,229-301$.

Toutain, J. Le deuxième congrès international d'archéologie classique. Alexandrie - Le Caire (7 avril 15 avril 1909). Journal des Savants, Juin 1909, 274-281.

Ungern-Sternberg, J. von. Politik und Geschichte. Der Althistoriker Eduard Meyer im Ersten Weltkrieg, in: Calder III - Demandt, 1990, 484-504.

Ungern-Sternberg, J. von. Ernst von Stern über Catilina und die Gracchen. Hyperboreus 2015, 21 281-304.

Welwei, K.-W. Das klassische Athen. Demokratie und Machtpolitik im 5. und 4. Jahrhundert. Darmstadt, 1999.

Zimmermann, H.-D. Eduard Meyer als hallescher Professor, in: Eduard Meyer (1855-1930), 1991, 9-15.

For citation: Ungern-Sternberg J. von. Wissenschaft und Zeitgeschichte: Die Briefe Ernst von Sterns an Eduard Meyer (1894-1918). Philologia Classica 2016, 11(1), 116-130. DOI: 10.21638/11701/spbu20.2016.111

\section{НАУКА И ИСТОРИЯ: ПИСЬМА ЭРНСТА ФОН ШТЕРНА ЭДУАРДУ МЕЙЕРУ (1894-1918)}

\section{Юрген бон Унгерн-Штернберг}

Сохранилось 18 писем, которые Эрнст фон Штерн, профессор древней истории в универитетах Одессы и Галле, написал выдающемуся историку Эдуарду Мейеру между 1894 и 1918 годами. Письма содержат разнообразные любопытные замечания, касающиеся вопросов древней истории и авторского права в России. В большей степени, однако, эти письма интересны как историческое свидетельство о событиях критического периода русской истории: переписка Штерна и Мейера затрагивает события первой русской революции, а также переговоры в Брест-Литовске в январе 1918 года.

Ключевые слова: древняя история, русские антиковеды, революция 1905 года, авторское право в России, Брест-Литовск. 